\title{
Electron-molecule collisions at low and intermediate energies using the R-matrix method
}

\author{
J. D. Gorfinkiel ${ }^{1}$, A. Faure ${ }^{2}$, S. Taioli ${ }^{1}$, C. Piccarreta ${ }^{1}$, G. Halmová $^{1}$, and J. Tennyson ${ }^{1}$ \\ 1 Department of Physics and Astronomy, University College London, Gower Street, London WC1E 6BT \\ ${ }^{2}$ Laboratoire d'Astrophysique, Observatoire de Grenoble, B.P. 53, 38041 Grenoble cedex 09, France
}

Received: date / Revised version: date

\begin{abstract}
We present the latest developments of the R-matrix method as applied to electron-molecule collisions. A variety of calculations for $\mathrm{H}_{2} \mathrm{O}$ are presented including the study of rotational excitation and preliminary data for dissociative electron attachment. Results for the application of the recently developed molecular R-matrix with pseudostates (MRMPS) method to neutral and cationic targets are also included. This method is currently being applied to the study of collisions with anionic targets.
\end{abstract}

PACS. PACS-key discribing text of that key - PACS-key discribing text of that key

\section{Introduction}

Electron-molecule collisions have been a field of active research over the last 25 years. Several theoretical methods have been developed (for a comprehensive summary, see [1]), mainly focused on the low energy regime. Specifically, elastic collisions, rotational, vibrational or electronic excitation and dissociative recombination and attachment are regularly investigated for small molecular targets. Among these methods, the R-matrix approach is one of the most successful.

Initially developed in the 1940s [2] to treat nuclear reactions, the R-matrix method was adapted to the study of electronatom collisions in the early 1970s [3]. A few years later [4, 5] the first applications to molecules were produced. However, it was not until the early 1990s that implementations of the method capable of treating collisions with polyatomic molecules were developed by the Bonn [6] and UK [7,8] groups. Their success has spurned more recent implementations $[9,10]$.

The UK molecular R-matrix codes have been applied to the study of the following processes: rotational excitation (within the adiabatic nuclear rotation model, e.g. $\mathrm{H}_{3}^{+}$and $\mathrm{H}_{3} \mathrm{O}^{+}$[11]), vibrational excitation (for diatomic molecules both in the adiabatic and non-adiabatic approximation: e.g. $\mathrm{NO}^{+}[12]$ ), electronic excitation (within the fixed-nuclei approximation: e.g. $\mathrm{CF}_{3}[13]$ and $\mathrm{SF}_{2}$ [14]) and electron impact dissociation (using the energy balancing method: $\mathrm{H}_{2}$ [15] and in one dimension for $\mathrm{H}_{2} \mathrm{O}$ [16]). The R-matrix method has also been used to provide resonance information to carry out dissociative recombination (DR) studies for $\mathrm{CO}^{2+}[17]$ and $\mathrm{NO}^{+}[18]$ and for a complete DR calculation in the case of $\mathrm{HeH}^{+}$[19].

We present in this paper results at the forefront of the application of the R-matrix method to molecules. These include calculations on electron impact rotational excitation of $\mathrm{H}_{2} \mathrm{O}$, molecular R-matrix with pseudostates (MRMPS) studies of $\mathrm{H}_{2}$ and $\mathrm{H}_{3}^{+}$and preliminary results on dissociative electron attachment (DEA) of water and collisions with $\mathrm{C}_{2}$.

The theory underlying molecular R-matrix calculations has been described in detail elsewhere (see $[7,8,20]$ and references therein). We will therefore not discuss it here. The paper is organized as follows: in section 2 we present our results for rotational excitation and DEA of $\mathrm{H}_{2} \mathrm{O}$. Section 3 introduces the MRMPS method and presents results for collisions with neutrals and positive and negative molecular ions. Finally, a summary and discussion of future perspectives is presented in section 4.

\section{Electron collisions with $\mathrm{H}_{2} \mathrm{O}$}

In the last two decades, collisions with water have been studied extensively, both experimentally and theoretically (for a review, see [21]). They are amongst the most extensively studied using R-matrix method. This is unsurprising: $\mathrm{H}_{2} \mathrm{O}$ is present in a great variety of environments (earth's atmosphere, astrophysical environments and more significantly, as the main constituent of living organisms). Collisions of electrons with water therefore play a crucial role in a great variety of research fields.

Among our results, those for rotational excitation have provided the best agreement with experiment to date. Differential cross sections (DCS) for the vibrationally elastic scattering have been computed and measured by several authors for collision energies below $50 \mathrm{eV}[22,23]$. Elastic DCS of water indeed provide crucial parameters for modelling the radiation effects on biological matter. Furthermore, rotationally inelastic integral cross sections (ICS) are required for modelling water line emission in various astronomical environments, notably cometary comae. To complement the work we have already 
performed, we have recently undertaken the full dimensional study of dissociative electron attachment of water.

Both calculations presented here are based on the work previously performed in the group [16] on electron impact dissociation. In that work, fixed-nuclei T-matrices were obtained for several geometries of $\mathrm{H}_{2} \mathrm{O}$. A detailed description of the basis sets used, and the configuration interaction models tested for the description of the electronic wavefunctions can be found there.

The rotational excitation calculations were performed using the ground state equilibrium geometry $(\mathrm{OH}$ bond length of $1.81 a_{0}$ and bond angle of $104.5^{\circ}$ ) wavefunctions generated using an $R$-matrix sphere of radius $10 a_{0}$. For this geometry, the molecule belongs to the $\mathrm{C}_{2 v}$ point group. The total $(\mathrm{N}+1)$ wavefunction was based on a close-coupling expansion that included the 7 lowest electronic states of water (model (a) in $[16]):{ }^{1} \mathrm{~A}_{1}(\tilde{X})$ (ground state), ${ }^{3} \mathrm{~B}_{1},{ }^{1} \mathrm{~B}_{1}(\tilde{A}),{ }^{3} \mathrm{~A}_{1},{ }^{1} \mathrm{~A}_{1}(\tilde{B})$, ${ }^{3} \mathrm{~B}_{2}$ and ${ }^{1} \mathrm{~B}_{2}$. The wavefunctions for these states were generated using averaged natural orbitals and a CASCI (complete active space configuration interaction) model. This model gives a ground-state energy of -76.0923 Hartree (in good agreement with the result of van Harrevelt and van Hemert [24]: -76.2905 Hart and a dipole moment of $1.864 \mathrm{D}$, which is close to the experimental value of $1.854 \mathrm{D}$ [25].

For the DEA calculations, the full dimensional potential energy surfaces corresponding to the three lowest resonances of water are required. For this reason a 9 state expansion was used. In the case of an arbitrary geometry, the water molecule belongs to the $\mathrm{C}_{s}$ point group. Using the notation for the irreducible representations of this point group, the nine states included were (model (b) in [16]): ${ }^{1} \mathrm{~A}^{\prime}(\tilde{X}), 1^{3} \mathrm{~A}^{\prime},{ }^{1} \mathrm{~A}^{\prime \prime}(\tilde{A}), 1^{3} \mathrm{~A}^{\prime}$, ${ }^{1} \mathrm{~A}^{\prime}(\tilde{B}), 2^{3} \mathrm{~A}^{\prime},{ }^{1} \mathrm{~A}^{\prime}\left(\tilde{E}^{\prime}\right)$ (already included included in the 7 state model) plus the $2^{3} \mathrm{~A}^{\prime \prime}$ and ${ }^{1} \mathrm{~A}$ ". Since these last two states are fairly diffuse, an R-matrix radius of $13 a_{0}$ was necessary in these calculations.

In both cases the close-coupling expansion was augmented with terms representing correlation and polarization. The continuum functions were represented by Gaussian-type orbitals (GTOs) optimized to represent Bessel functions, with $l \leq 4$ [26]; a different set of GTOs was used for each radius.

\subsection{Electron-impact rotational excitation}

Differential cross sections (DCS) were calculated following the procedure implemented in the program POLYDCS [27] as recently employed in [28], using the electron- $\mathrm{H}_{2} \mathrm{O}$ wavefunctions for the equilibrium geometry of $\mathrm{H}_{2} \mathrm{O}$ calculated as described above. The general theory of the scattering of an electron from a polyatomic molecule in the fixed-nuclei (FN) approximation has been presented in detail elsewhere (see, e.g., [29]). In this approach, the DCS is expressed as a partial-wave expansion within the adiabatic-nuclei-rotation (ANR) approximation which assumes that the initial and final target states are degenerate. For low partial-waves (here $l \leq 4$ ), the DCS is computed from the FN $T$-matrices obtained via the $R$-matrix calculations. In the case of a polar molecule, the partial-wave expansion does not converge in the FN approximation, owing to the very long-range nature of the electron-dipole interaction. To circumvent this problem, the standard procedure is to use

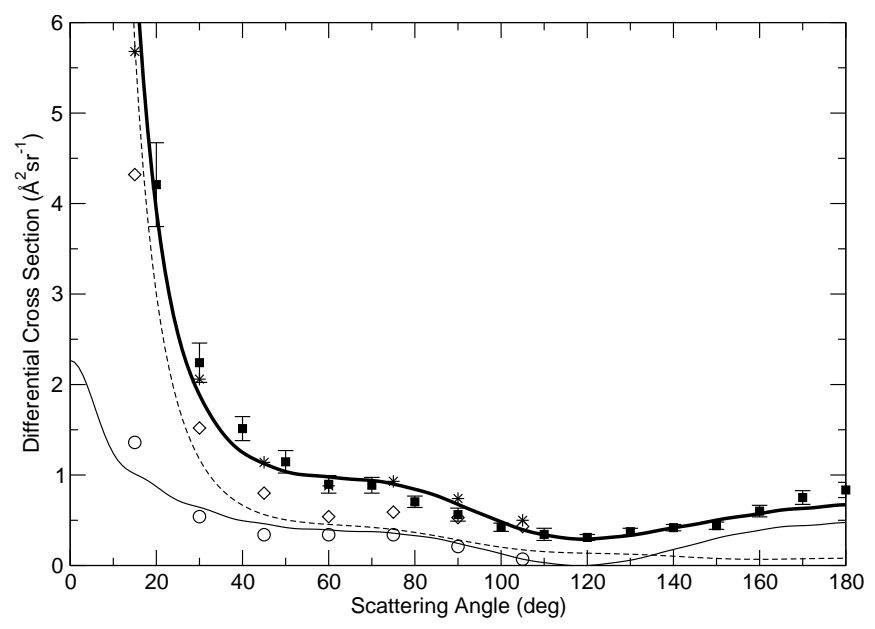

Fig. 1. Computed and measured DCS of water at $6 \mathrm{eV}$. The present elastic (rotationally summed) DCS is given by the thick solid line. Other lines denote partial state-to-state DCS (dashed line: $0 \rightarrow 1$; solid line: $0 \rightarrow 0$ ). The filled squares correspond to the experimental elastic DCS of [22]. The open circles and diamonds correspond rerenectively to the experimental pure elastic $(\Delta j=0)$ and rotationally inelastic $(\Delta j= \pm 1)$ DCS of [30]; the sum of both contributions is given by the stars.

the dipolar Born approximation to obtain the DCS for the high partial-waves not included in the FN $T$-matrices. The final DCS is then calculated as the sum of two contributions and can be regarded as a short-range correction to the Born approximation.

Figure 1 compares our results at $6 \mathrm{eV}$ with the rotational excitation measurements of Jung et al. [30] and the elastic measurements of Cho et al. [22]. All calculations were performed for the water molecule in its ground rotational state $(j \tau)=$ (00). As shown by Okamoto et al. [31], the elastic (rotationally summed) DCS does indeed not depend on the initial rotational state of the water molecule unless the scattering angle is very small (less than $0.1^{\circ}$ at $6 \mathrm{eV}$ ) or the collisional energy is very low (close to rotational thresholds). Furthermore, when summed over the final $\tau^{\prime}$ pseudo-quantum number, the dominant partial contributions to the elastic DCS $(\Delta j=0, \pm 1$ and \pm 2 , see [28]) only weakly depend on the initial rotational state. DCS calculations for the $(00) \rightarrow(00)$ and $(00) \rightarrow(10)$ transitions are therefore directly comparable to the $\Delta j=0, \pm 1$ measurements of Jung et al. performed at $450 \mathrm{~K}$. A proper averaging over the experimental rotational distribution would prove necessary only at very low collision energy.

In fig. 1, we can first notice the very good agreement with the elastic measurements of Cho et al. over the whole measured angular range. The partial DCS are plotted in the same figure. The agreement with the data of Jung et al. is quite satisfactory (within 50\%) in view of the experimental and theoretical uncertainties. In particular, it should be noted that the sum of the Jung et al.'s DCS is in excellent agreement with the present rotationally summed DCS. Therefore, the small discrepancies between the experimental and theoretical partial DCS probably reflect the contribution of $\Delta j=2$ transitions which were ignored in the experimental fitting procedure. Finally, we note that the elastic ICS was also determined by Cho et al. by extrapolating the experimental DCS at forward angles. Such ex- 
trapolation procedure introduces rather large uncertainties because the large dipole moment of water leads to heavily forward peaked DCS [28]. As a result, the experimental elastic ICS was found to be lower than the theoretical one by a factor of 2.4 at $6 \mathrm{eV}$.

\subsection{Dissociative electron attachment}

The recent increase in computer power and the development of new numerical methods [32] makes it possible to use a timedependent (TD) treatment to study many phenomena like photodissociation [33], reactive collisions [34], BEC [35] and femtoseconds laser pulse [36]. Because evolution in time is the natural frame for dynamics and the energy and time domain are linked by a simple Fourier transform, we will employ a timedependent method for calculating the nuclear dynamics subsequent to electron impact. This method allows the treatment of the full multidimensional interplay between multiple nuclear degrees of freedom and non-adiabatic open (i.e. energetically accessible) non-resonant decay processes.

Experimental studies of DEA of water include those of Melton [37], Belic̀ et al. [38] and Curtis et al. [39] and more recently Harb et al. [40]. A very recent theoretical work [41] employed the Complex Kohn variational and a similar approach to ours. However, these authors only considered the first resonance of the system (labelled ${ }^{2} \mathrm{~B}_{1}$ in $\mathrm{C}_{2 v}$ notation) whereas our aim is to take into account the three lowest resonances (the above mentioned and the ${ }^{2} \mathrm{~A}_{1}$ and ${ }^{2} \mathrm{~B}_{2}$ ). Furthermore, in our work all resonance parameters (positions and widths or lifetimes) are being determined consistently by means of collisional calculations. To obtain an accurate description of the resonance surfaces, we performed calculations for 840 geometries of $\mathrm{H}_{2} \mathrm{O}$. These geometries were obtained by varying the internal nuclear coordinates of the molecule along the following values: $14 \mathrm{rOH}_{\mathrm{OH}}$ internuclear distances in the range $(1.3-2.6) \mathrm{a}_{0}$ with a step of $0.1 \mathrm{a}_{0}$ and 8 different $\vartheta$ internal angles $\left(70^{\circ}, 80^{\circ}, 90^{\circ}, 104.5^{\circ}\right.$, $120^{\circ}, 130^{\circ}, 150^{\circ}, 170^{\circ}$ ).

Figure 2 shows resonance positions and widths for the two lowest resonances calculated for $\vartheta=104.5^{\circ}$ and $\vartheta=90^{\circ}$ and $r_{\mathrm{OH} 1}$ that is, the symmetric stretching of both $\mathrm{OH}$ bonds. Full resonance surfaces will be published elsewhere. For both angles, the energy of the ${ }^{2} \mathrm{~B}_{1}$ resonance, whose leading configuration for the equilibrium geometry is $1 \mathrm{a}_{1}^{2} 2 \mathrm{a}_{1}^{2} 3 \mathrm{a}_{1}^{2} 1 \mathrm{~b}_{2}^{2} 1 \mathrm{~b}_{1}^{1} 4 \mathrm{a}_{1}^{2}$, follows closely that of its parent state ${ }^{3} \mathrm{~B}_{1}$. Similarly, the ${ }^{2} \mathrm{~A}_{1}$ resonance, with $1 a_{1}^{2} 2 a_{1}^{2} 3 a_{1}^{1} 1 b_{2}^{2} 1 b_{1}^{2} 4 a_{1}^{2}$ as the leading configuration for the equilibrium geometry, follows that of the ${ }^{3} \mathrm{~A}_{1}$ state. The corresponding widths decrease as the $r_{\mathrm{OH}}$ distances increase, but the ${ }^{2} \mathrm{~B}_{1}$ resonance has its maximum width for $r_{\mathrm{OH}}=1.5 \mathrm{a}_{0}$; for this resonance the widths for both angles are very similar. A cut of the surfaces corresponding to stretching a single bond can be found in [16].

\section{Molecular R-matrix with pseudostates method}

Standard close-coupling expansions (as those used in the standard R-matrix method, the Kohn variation method, etc.) are

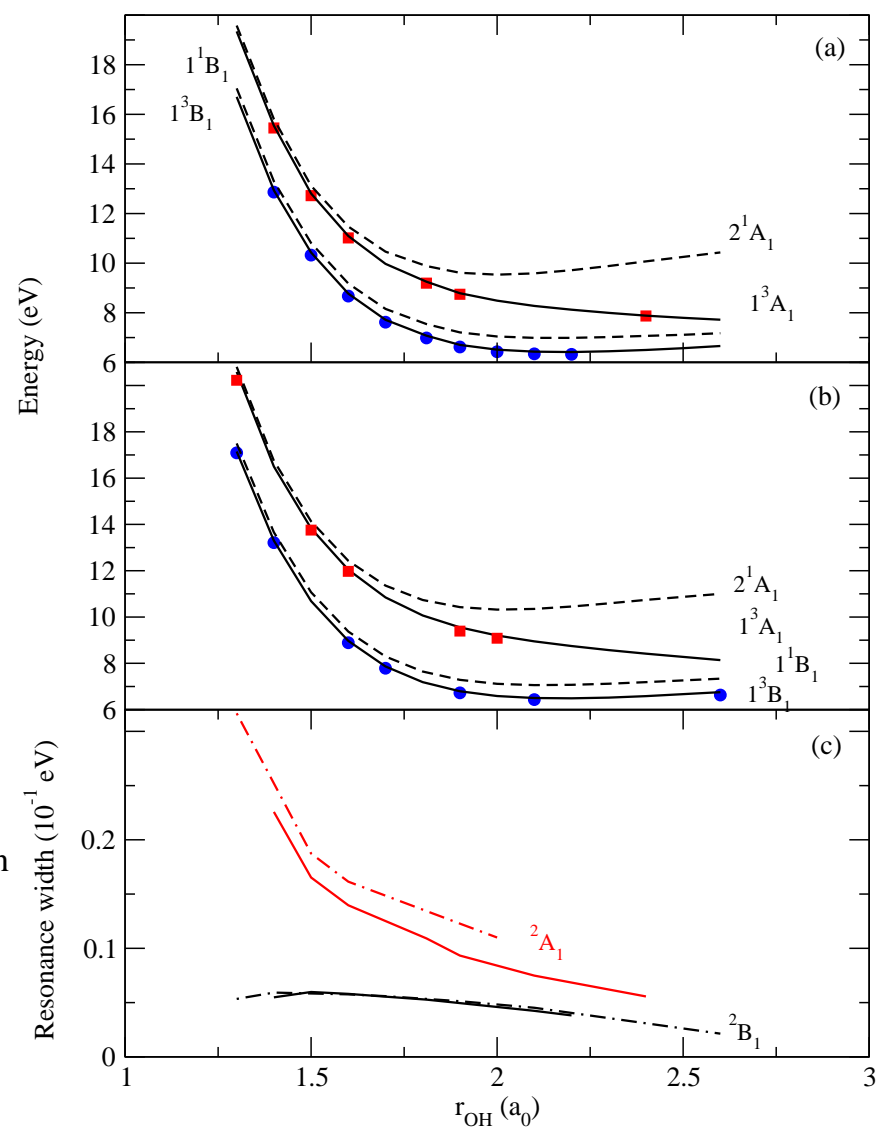

Fig. 2. Resonance positions and widths as a function of the $\mathrm{r}_{\mathrm{OH}}=\mathrm{r}_{\mathrm{OH} 1}=\mathrm{rOH}_{\mathrm{O}}$ bond length for (a) $\vartheta=104.5^{\circ}$ and (b) $\vartheta=90^{\circ}$ for the ${ }^{2} \mathrm{~B}_{1}$ and ${ }^{2} \mathrm{~A}_{1}$ resonances. In (a) and (b) the curves correspond to the threshold energies of the four lowest excited states (full curves: triplets, dashed curves: singlets) and the circles and squares to the ${ }^{2} \mathrm{~B}_{1}$ and ${ }^{2} \mathrm{~A}_{1}$ resonance positions respectively. Widths plotted in panel (c): full lines, $\vartheta=104.5^{\circ}$ and dotted-dashed lines, $\vartheta=90^{\circ}$.

inherently incomplete: they cannot include all excited target states and they do not attempt to incorporate the continuum of $\mathrm{PPH}^{2}$ target. Only the Convergent Close-Coupling method [42] can in principle ensure completeness, and only for one active electron systems, by using Sturmian functions as basis functions. This incompleteness can limit the quality of the description of low-energy processes (for example, because of the crude representation of the polarization) and also prevents these methods from being applicable at intermediate energies, i.e. those collision energies close to and above the ionization threshold.

We have recently developed and implemented [43] a molecular R-matrix with pseudostates (MRMPS) method. This method, based on the atomic R-matrix with pseudostates method [44] has allowed us to produce $a b$ initio cross sections for nearthreshold ionisation as well as electronic excitation. The first ionisation threshold can be very low for molecules and therefore the MRMPS method could be necessary even below $10 \mathrm{eV}$. The method is fully general and a detailed description of it can be found in [45].

Briefly, the MRMPS method is based on the use of some states that represent a discretized continuum. These states, known as pseudostates, are eigensolutions of the molecular Hamilto- 
Table 1. Symmetry, resonance position $\left(E_{r}\right.$ in $\left.\mathrm{eV}\right)$ and resonance width $\left(\Gamma_{r}\right.$ in $\left.\mathrm{eV}\right)$ at the equilibrium geometry of $\mathrm{H}_{3}^{+}$. Column A corresponds to an MRMPS calculation with 64 states; column B corresponds to a standard 6 state calculation (using $\mathrm{H}_{3}^{2+}$ orbitals). Columns C are corrected results from [48] (see text).

\begin{tabular}{ccccccc}
\hline Symm. & \multicolumn{2}{c}{$\mathrm{A}$} & \multicolumn{2}{c}{$\mathrm{B}$} & \multicolumn{2}{c}{$\mathrm{C}$} \\
\hline $\mathrm{D}_{3 h}$ & $E_{r}$ & $\Gamma_{r}$ & $E_{r}$ & $\Gamma_{r}$ & $E_{r}$ & $\Gamma_{r}$ \\
\hline${ }^{2} \mathrm{E}^{\prime}$ & 8.74 & 0.60 & 8.66 & 0.59 & 9.19 & 0.64 \\
${ }^{2} \mathrm{~A}_{1}$ & 9.62 & 0.18 & 9.56 & 0.17 & 10.17 & 0.18 \\
${ }^{2} \mathrm{~A}_{2}$ & 10.79 & 0.0006 & - & - & 11.17 & 0.0006 \\
${ }^{2} \mathrm{E}^{\prime}$ & 10.86 & 0.10 & 10.63 & 0.084 & 11.33 & 0.090 \\
${ }^{2} \mathrm{E}$, & 10.97 & 0.098 & 10.62 & 0.11 & 11.26 & 0.098 \\
${ }^{2} \mathrm{E}$, & 12.74 & 0.080 & 12.33 & 0.072 & 12.95 & 0.078 \\
${ }^{2} \mathrm{~A}_{1}^{\prime}$ & 12.87 & 0.026 & 12.45 & 0.022 & 13.07 & 0.025 \\
${ }^{2} \mathrm{E}^{\prime}$ & 13.00 & 0.013 & 12.56 & 0.011 & 13.21 & 0.015 \\
${ }^{2} \mathrm{E}^{\prime}$ & 13.05 & 0.030 & 12.62 & 0.025 & 13.27 & 0.027 \\
${ }^{2} \mathrm{~A}^{\prime}$ & - & - & 12.62 & 0.016 & - & - \\
\hline
\end{tabular}

nian within the basis set used but do not represent real eigenstates of the system. If chosen carefully, they give a proper description of the continuum states of the target at short range. Due to the reduced symmetry of the problem, each of these pseudostates is associated with several asymptotic channels. The pseudostates are included in the close-coupling expansion used to represent the basis state wavefunctions for the target plus electron in the inner region. Transition into the pseudostates that are above the ionization threshold can be interpreted as ionization. In practise, a projection technique may be required to extract the bound component from the pseudostates.

The key point of the method lies in how to generate a set of pseudostates that ensures a good description of the ionized target. In our implementation, we use GTOs as basis functions for all (bound, continuum and pseudocontinuum) orbitals. We have chosen to use an even-tempered basis set for the pseudocontinuum orbitals, so their exponents follow: $\alpha_{i}=\alpha_{0} \beta^{(i-1)}$. However, no systematic strategy for the choice of $\alpha_{0}$ and $\beta$ has yet been found.

\subsection{Positive ions}

The $\mathrm{H}_{3}^{+}$molecule is the dominant ion in low-temperature hydrogenic plasmas. Its interaction with low-energy electrons has been widely studied, but until very recently no information was available on intermediate energies (experiments on $\mathrm{D}_{3}^{+}$have been performed [46] but are yet unpublished). The MRMPS method has been applied to $\mathrm{H}_{3}^{+}$[43] and resonance parameters and electronic excitation and ionisation cross sections have been determined. Our pseudostates span the ionisation threshold of $\mathrm{H}_{3}^{+}$, which lies at the relatively high value of $33.47 \mathrm{eV}$.

One of the problems of the inclusion of pseudostates in the close-coupling expansion is the appearance of pseudo-resonance These are non-physical resonances whose parent state is one of the pseudostates. This problem is particularly severe for positive ions, where an infinite number of Rydberg states of the $\mathrm{N}+1$ system converge to the different excitation thresholds. To overcome this problem, we implemented a convolution plus averaging technique based on the ideas of Meyer et al. [47]. This technique was applied to $\mathrm{H}_{3}^{+}$and provided fairly smooth results for ionisation. Its limitation lies in the fact that it eliminates all resonances, including physical ones. For this reason, the method cannot be applied below the ionisation threshold, where resonances are physical. In the same way, if physical resonances are present above the ionisation threshold, they will also be eliminated.

The calculated cross sections for excitation into the first two excited states of $\mathrm{H}_{3}^{+}$were in good agreement with previous calculations $[48,49]$. Since the Faure and Tennyson calculation [48] was performed, a bug affecting the treatment of degenerate states has been corrected in the R-matrix suite. Furthermore, the resonance parameters published corresponded to a model in which $5 \mathrm{a}_{1}, 1 \mathrm{~b}_{1}, 3 \mathrm{~b}_{2}$ and no $\mathrm{a}_{2} \mathrm{H}_{3}^{+}$molecular orbitals were used (instead of the 5,3,3,1 mentioned in the paper). These calculations showed an unphysical splitting of the two components of degenerate $\left({ }^{2} E\right.$ ' and $\left.{ }^{2} E^{\prime \prime}\right)$ resonances.

For this reason, we re-ran the calculation (with a 5,3,3,1 model). The size and shape of the cross section did not change significantly, but the resonance parameters did. In Table 1, the positions and widths of the 9 lowest lying resonances are presented for 3 calculations: A) an MRMPS one in which 64 bound and pseudostates were included (see details in [45]), B) a standard 6 state calculation (both A and B used molecular orbitals for $\mathrm{H}_{3}^{2+}$ ) and C) a re-run of the calculations from [48]. Orel and Kulander [50] reported the following positions and widths (in $\mathrm{eV}$ ) for the two lowest resonances: 9.1, 0.64 and 10.3, 0.18. Differences between the results of $\mathrm{B}$ and $\mathrm{C}$ are mainly due to the shift in excitation thresholds. However, for the A and B results an examination of the quantum defects shows a consistent increase in the value of $\alpha$ for the 64 state calculation (A). We attribute this increase to the correct representation of the polarization in the MRMPS calculation. Indeed, inclusion of pseudostates in the calculation produced polarization values within $2 \%$ of very accurate results. Standard close-coupling expansions, in contrast, do not produce converged polarizabilities.

\subsection{Neutrals}

Recently, we performed some initial calculations for ionization of $\mathrm{H}_{2}$ [45]. This is a benchmark system and a variety of experimental and theoretical data are available. The problem when treating $\mathrm{H}_{2}$ is the diffuse quality of its low-lying excited states. An R-matrix box with a radius of $a=20 \mathrm{a}_{0}$ is required [51] to contain these states, making calculations computationally very demanding. For this reason, we chose to represent accurately only the (compact) ground and first excited states. The remaining states were treated as pseudostates thus allowing us to use a radius $a=10 \mathrm{a}_{0}$.

No convolution plus averaging procedure was necessary for this system as no pseudo-resonances were visible in the (unaveraged) ionisation cross sections. Two MRMPS results are shown in figure 3: those for a partial wave expansion with $l \leq 3$ and for $l \leq 4$. It can be seen that the results are converged up to $5 \mathrm{eV}$ above the ionisation threshold (whose value is $15.4 \mathrm{eV}$ for the adiabatic case and $16.4 \mathrm{eV}$ for the vertical case) and differences for $26 \mathrm{eV}$ are about $20 \%$. A method to top-up the partial wave expansion (for example, as used in atomic R-matrix with pseudostates calculations [52]) will be needed if cross sections 


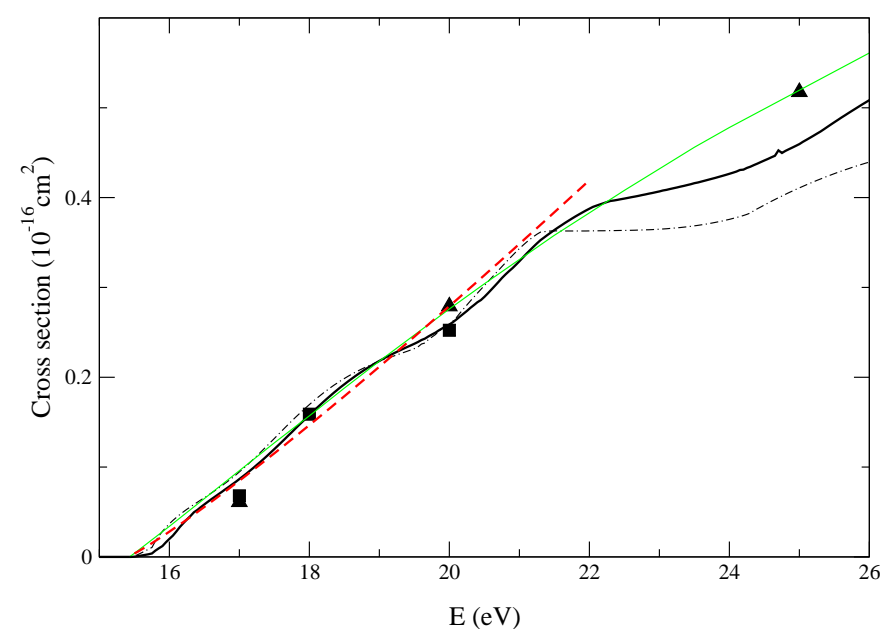

Fig. 3. Ionisation cross section for $\mathrm{H}_{2}$. Full dark line: MRMPS results with $l \leq 4$ [45], dotted-dashed line: MRMPS results with $l \leq 3$, light line: Binary-Encounter-Bethe semi-analytical model [55], dashedline: Wannier law, $\sigma \propto \mathrm{E}^{1.1269}$ Experimental results: squares, Krishankumar et al. [53] and triangles, Straub et al. [54].

are to be calculated for higher energies. As shown in figure 3, the resulting averaged cross section is in excellent agreement with experimental results $[53,54]$ almost up to $15 \mathrm{eV}$ above the ionisation threshold . It also agrees very well with the BinaryEncounter-Bethe semi-analytical model [55] and the cross section obtained from application of the Wannier law [56], up to $4 \mathrm{eV}$ above the ionisation threshold.

\subsection{Negative ions}

Storage ring experiments which studied low-energy electron collisions with diatomic molecular anions, including $\mathrm{C}_{2}^{-}$, showed rather unexpected resonance features [57,58]. Since these resonances lie above the (low) ionisation energy of the anions, they are not amenable to study with standard close-coupling techniques. Our plan is to use the MRMPS method to study these systems.

As a precursor to studying electron collisions with $\mathrm{C}_{2}^{-}$, we have performed a study of bound and continuum states of $\mathrm{C}_{2}^{-}$ itself, which involves calculations of electron collisions with $\mathrm{C}_{2}$. Such collisions are of interest in their own right since the $\mathrm{C}_{2}$ molecule is well known in flames, astrophysically and could be important at the edge of fusion plasmas. $\mathrm{C}_{2}$ is actually a rather complicated system with many electronically states within a few $\mathrm{eV}$ of its $\mathrm{X}^{1} \Sigma_{g}^{+}$ground state [59].

Electron collision calculations were performed for with $\mathrm{C}_{2}$ frozen at its equilibrium geometry of $1.2425 \AA$. Various closecoupling models were tested, the largest of which, for which results are reported here, used 26 target states in the close coupling expansion. The states were represented using natural orbitals and a CASCI model in which the four 1s electrons were frozen in the $1 \sigma_{g}$ and $1 \sigma_{u}$ orbitals, and the remaining eight electrons were distributed freely among the $2 \sigma_{g}, 3 \sigma_{g}, 2 \sigma_{u}, 3 \sigma_{u}, 1$ and $1 \pi_{g}$ orbitals, subject only to the constraint of the overall space and spin symmetry of the final state. Calculations were performed for an R-matrix radius of $a=10 \mathrm{a}_{0}$ and repeated

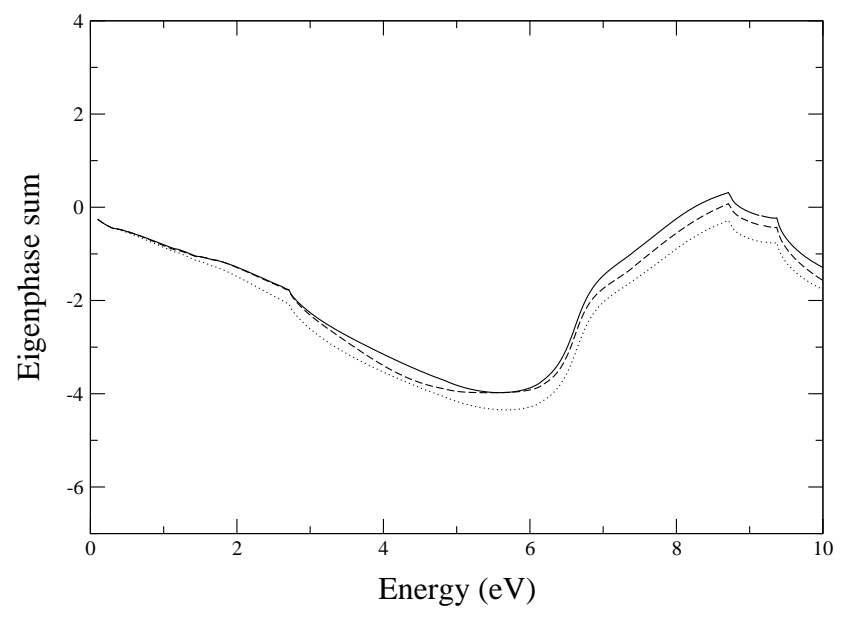

Fig. 4. Calculated ${ }^{2} \mathrm{~A}_{g}\left({ }^{2} \Sigma_{g}^{+}+{ }^{2} \Delta_{g}+\ldots\right)$ eigenphase sums for lowenergy electron collisions with $\mathrm{C}_{2}$. Solid line: $a=10 \mathrm{a}_{0}$ and basis of Faure et al [26]; dashed line: $a=13 \mathrm{a}_{0}$ and basis of Faure et al [26]; dotted line: $a=10 \mathrm{a}_{0}$ and basis of Sarpal et al [60].

for $a=13 \mathrm{a}_{0}$, and a variety of continuum basis sets [26,60]. Figure 5 gives a comparison of the eigenphases obtained for the totally symmetric irreducible representation, $\mathrm{A}_{g}$ in the $\mathrm{D}_{2 h}$ point group used for the calculations. The eigenphases show good agreement between the various models. The best calculation corresponds to that with $a=10 \mathrm{a}_{0}$ and basis of Faure et al [26]. This model was therefore used to look for bound states of the $\mathrm{C}_{2}^{-}$system.

Table 2 compares our results with a number of studies in the literature. Our data are for a single frozen bondlength, that is for vertical transitions, and corresponds to the ionization potential and excitation thresholds of $\mathrm{C}_{2}^{-}$. Previous results correspond to the adiabatic process and hence their ionisation potential can also be interpreted (with a sign change) as the electron affinity of $\mathrm{C}_{2}$. Given that our data are vertical whereas the other work all considers adiabatic energies, the agreement can be considered to be very satisfactory.

Huber and Herzberg [59] also report tentative experimental evidence for a ${ }^{4} \Sigma_{u}^{+}$bound state of $C_{2}^{-}$[61]. However, our calculations showed no evidence for any other bound state, of either doublet or quartet spin symmetry. Given the reliability of our results for the doublet states, we consider it unlikely that there are any strongly bound states of $\mathrm{C}_{2}^{-}$with quartet spin symmetry.

\section{Conclusions}

The R-matrix method has proved to be a flexible tool for treating a variety of problems involving electron collisions with molecules. It can be used for studies ranging from the characterisation of diffuse bound states to near threshold impact ionisation, using the newly developed [43] molecular R-matrix $\pi$ with pseudostates (MRMPS) method. For low-energy collisions the vibrational and rotational motions of the nuclei can also be modelled. Such calculations have been demonstrated to give an accuracy rivalling, and sometimes exceeding [28] experiment. 
Table 2. Bound states of $\mathrm{C}_{2}^{-}$obtained from scattering calculations (this work), via various electronic structure methods and observed experimentally. The ionization potential (IP) is provided for the ground state and the excitation threshold $\left(\mathrm{T}_{e}\right)$ for the excited states.

\begin{tabular}{llccc}
\hline & & $\mathrm{X}^{2} \Sigma_{g}^{+}$ & $\mathrm{A}^{2} \Pi_{u}$ & $\mathrm{~B}^{2} \Sigma_{u}^{+}$ \\
\hline MRD-CI [62] & $R_{e} / \AA$ & 1.2780 & 1.3180 & 1.2220 \\
& $\mathrm{IP}^{+} \mathrm{T}_{e} / \mathrm{eV}$ & $\sim 3.4$ & 0.403 & 2.335 \\
MCSCF [63] & $R_{e} / \AA$ & 1.2760 & 1.3180 & 1.2310 \\
& $\mathrm{IP}^{\mathrm{T}} \mathrm{T}_{e} / \mathrm{eV}$ & $\sim 3.3$ & 0.435 & 2.348 \\
QCISD [64] & $R_{e} / \AA$ & 1.2775 & & \\
& $\mathrm{IP}^{\mathrm{T}} \mathrm{T}_{e} / \mathrm{eV}$ & 2.74 & & \\
CCSD [65] & $R_{e} / \AA$ & 1.2670 & 1.3070 & 1.2220 \\
& $\mathrm{IP}^{\mathrm{T}} \mathrm{T}_{e} / \mathrm{eV}$ & 3.09 & 0.553 & 2.453 \\
This work & $R_{e} / \AA$ & 1.2425 & 1.2425 & 1.2425 \\
& $\mathrm{IP}^{\mathrm{T}} \mathrm{T}_{e} / \mathrm{eV}$ & 3.04 & 0.722 & 2.131 \\
Obs. [59] & $R_{e} / \AA$ & 1.26821 & & 1.2233 \\
& $\mathrm{IP}, \mathrm{T}_{e} / \mathrm{eV}$ & & & 2.280 \\
\hline
\end{tabular}

The calculations reported here are all for small target molecu Some calculations have been performed for large systems such as the electron rich $\mathrm{CF}_{3}$ radical [13], and the small organic molecule cyclopropane [66]. However it is clear that the next major challenge for such studies is their extension to electron collision from medium sized, or even large, molecules of interest.

\subsection{Acknowledgement}

We gratefully acknowledge the support of the EPIC network. This work was also funded by the EPSRC. Most of the calculations were carried out on the Ra Supercomputer, at the HiPerSPACE Computing Centre at UCL. DCS calculations for water were performed on the workstations of the "Service Commun de Calcul intensif de l'Observatoire de Grenoble". AF acknowledges the support of the CNRS.

\section{References}

1. Huo W M and Gianturco F A, eds Computational Methods for Electron-Molecule Collisions (Plenum Press 1995).

2. Wigner E P and Eisebud L, Phys. Rev. 72, (1947) 29.

3. Burke P G, Hibbert A and Robb W D, J. Phys. B 4, (1971) 153.

4. Schneider B I, Chem. Phys. Lett. 31, (1975) 237; Schneider B I, Phys. Rev. A 11, (1975) 1957.

5. Burke P G, Mackey I and Shimamura I, J. Phys. B 10, (1977) 2497.

6. Pfi ngst K, Nestmann B M and Peyerimhoff S D, J. Phys. B 27, (1994) 2283.

7. Morgan L A, Gillan C J, Tennyson J and Chen X, J. Phys. B 30, (1997) 4087

8. Morgan L A, Tennyson J and Gillan C J, Computer Phys. Comms. 114, (1998) 120.

9. Huo W M and Brown D, Phys. Rev. A 60, (1999) 295.

10. Tonzani T and Greene C H, J. Chem. Phys 122, (2005) 014111.

11. Faure A and Tennyson J, Mon. Not. R. Astron. Soc. 340, (2003) 468.

12. Rabadan I and Tennyson J, J. Phys. B 32, (1999) 4753.
13. Rozum I, Mason N J and Tennyson J, New J. Phys. 5, (2003) 1-155.

14. Baluja K L and Tossell J A, J. Phys. B 37, (2004) 609.

15. Trevisan C S and Tennyson J, J. Phys. B 34, (2001) 2935.

16. Gorfi nkiel J D, Morgan L A and Tennyson J, J. Phys. B. 35, (2002) 543

17. Vinci N, de Ruette N, Waffeu-Tamo F O, Motapon O, Fifi rig M, Crumeyrolle O, Urbain X, Tennyson J and Schneider I F, J. Phys.: Conference Series 4, (2005) 162.

18. Schneider I F, Rabadan I, Carata L, Tennyson T, Andersen L H and Suzor-Weiner A, J. Phys. B 33, (2000) 4849.

19. Sarpal B K, Tennyson J and Morgan L A, J. Phys. B. 27, (1994) 5943.

20. Tennyson J and Morgan L A, Phil. Trans. A 357, (1999) 1161.

21. Mason N J, Itikawa Y, in preparation.

22. Cho H, Park Y S, Tanaka H, Buckman S J, J. Phys. B 37, (2004) 4639.

23. Gianturco F A, Meloni S, Paioletti P, Lucchese R R and Sanna N, J. Chem. Phys. 108, (1998) 4002.

24. van Harrevelt R and van Hemert M C, J. Chem. Phys. 112, (2000) 5777.

25. Suresh S J, Naik V M, J. Chem. Phys 113, (2000) 9727.

26. Faure A, Gorfi nkiel J D, Morgan L A, Tennyson J, Computer. Phys. Commun. 144, (2002) 224.

27. Sanna N and Gianturco F A, Comput. Phys. Commun. 114, (1998) 142.

28. Faure A, Gorfi nkiel J D and Tennyson J, J. Phys. B. 37, (2004) 801.

29. Gianturco F A and Jain A, Phys. Rep. 143, (1986) 347.

30. Jung K, Antoni Th, Müller R, Kochem K H, Erhardt H, J. Phys. B. 15, (1982) 3535 .

31. Okamoto Y, Onda K and Itikawa Y, J. Phys. B. 26, (1993) 745.

32. Balakrishnan N, Kalyanaram C, Sathyamurthy N, Phys. Rep. 280, (1997) 79.

33. Tannor D J and Rice S A, Adv. Chem. Phys. 70, (1988) 441.

34. Zhang D H and Zhang J Z H, J. Chem. Phys 101, (1994) 3671.

35. Inguscio M, Stringari S, and Wieman C, Bose-Einstein condensation in atomic gases (IOS Press, Amsterdam, 1999).

36. Zewail A H, Femtochemistry (World Scientifi c, Singapore, 1994).

37. Melton C E, J. Chem. Phys. 57, (1972) 4218.

38. Belic̀ D S, Landau M and Hall R I, J. Phys. B 14, (1981) 175.

39. Curtis M G and Walker I C, J. Chem. Soc. Faraday Trans. 88, (1992) 2805.

40. Harb T, Kedzierski W and McConkey J W, J. Chem.Phys. 17, (2001) 123.

41. Haxton D J, Zhang Z, Meyer H D, Rescigno T N and McCurdy C W, Phys. Rev. A 69, (2004) 062714.

42. Bray I, Fursa D V, Kheifets A S and Stelvobics A T, J. Phys. B 35, (2002) R117.

43. Gorfi nkiel J D and Tennyson J, J. Phys B 37, (2004) L343.

44. Bartschat K, Hudson E T, Scott M P, Burke P G and Burke V M, J. Phys. B 29, (1996) 115.

45. Gorfi nkiel J D and Tennyson J, J. Phys. B, in press.

46. Ghazaly M E et al, in preparation.

47. Meyer K W, Greene C H and Bray I, Phys. Rev. A 52, (1995) 1334.

48. Faure A and Tennyson J, J. Phys. B 35, (2002) 1865.

49. Orel A E Phys. Rev. A 46, (1992)1333.

50. Orel A E and Kulander K C, Phys. Rev. Lett. 71, (1993) 4315.

51. Branchett S E and Tennyson J, Phys. Rev. Lett 64, (1990) 2889.

52. Bartschat K and Bray I, J. Phys. B 29, (1996) L577.

53. Krishnakumar E and Srivastava S K, J. Phys. B 27, (1994) L251. 
54. Straub H C, Renault P, Lindsay B G, Smith K A and Stebbings R F, Phys Rev. A 54, (1996) 2146.

55. Kim Y K and Rudd M E, Phys. Rev. A 50, (1994) 3954.

56. Wannier G H, Physical Review 90, (1953) 817.

57. Pedersen H B, Djuric N, Jensen M J, Kella D, Safvan C P, VejbyChristensen L, and Andersen L H, Phys. Rev. Lett. 81, (1998) 5302.

58. Pedersen H B, Djuric N, Jensen M J, Kella D, Safvan C P, Schmidt H T, Vejby-Christensen L, and Andersen L H, Phys. Rev. A 60, (1999) 2882.

59. Huber K P and Herzberg G, Constants of Diatomic Molecules (Van Nostrand Reinhold 1979).

60. Sarpal B K, Pfi ngst K, Nestmann B M and Peyerimhoff S D., J. Phys. B 29, (1996) 857.

61. Bondybey V E and Brus L E, J. Chem. Phys. 63, (1975) 2223.

62. Zeitz M, Peyerimhoff S D and Buemker R J, Chem. Phys. Lett. 64, (1979) 243.

63. Rosmus P and Werner H-J,J. Chem. Phys. 80, (1984) 5085.

64. Wang R, Zhu Z H and Yang C L, J. Mol. Struct. 571, (2001) 133.

65. Watts J D and Bartlett R J, J. Chem. Phys. 96, (1992) 6073.

66. Beyer T, Nestmann B M, Sarpal B K and Peyerimhoff S D. J. Phys. B 30, (1997) 3431. 